\title{
Fuzzy stochastic differential equations driven by fractional Brownian motion
}

\author{
Hossein Jafari ${ }^{*}$, Marek T. Malinowski ${ }^{2}$ and M.J. Ebadi ${ }^{1}$
}

"Correspondence: jafari@cmu.ac.ir

'Department of Mathematics, Chabahar Maritime University,

Chabahar, Iran

Full list of author information is

available at the end of the article

\begin{abstract}
In this paper, we consider fuzzy stochastic differential equations (FSDEs) driven by fractional Brownian motion ( $\mathrm{fBm}$ ). These equations can be applied in hybrid real-world systems, including randomness, fuzziness and long-range dependence. Under some assumptions on the coefficients, we follow an approximation method to the fractional stochastic integral to study the existence and uniqueness of the solutions. As an example, in financial models, we obtain the solution for an equation with linear coefficients.
\end{abstract}

Keywords: Fuzzy set theory; Fuzzy stochastic processes; Fuzzy stochastic differential equation; Fractional Brownian motion

\section{Introduction}

The FSDEs are used in real-world systems that the phenomena are related to randomness and fuzziness as two kinds of uncertainties, such as in economics and finance. There are papers on FSDEs that each one is different from the others in the approach. The author in [9] presented a definition of the fuzzy stochastic Itô integral. In [12-15] the author used the fuzzy non-anticipating stochastic processes and Wiener process to drive the fuzzy Itô stochastic integral. The method deals with the embedding of a crisp Itô stochastic integral into fuzzy space to build a fuzzy random variable.

On the other hand, in the modeling of many stochastic systems, the fBm which shows a long-range dependence, is suggested to replace the Brownian motion as the driving process. The fBm with $H \in(0,1)$ as Hurst parameter is a Gaussian process with beneficial properties, long-range dependence, self-similarity and stationary of increments. This process is appropriate for the analysis of phenomena which present long-range and scaleinvariant correlations. Nevertheless, when $H \neq \frac{1}{2}$, the $\mathrm{fBm}$ is not a semimartingale.

In this paper, we introduce FSDEs with respect to the $\mathrm{fBm}$. These equations can be helpful in the modeling of hybrid dynamic systems, including randomness, fuzziness and longrange dependence. We apply an approximation procedure to fractional stochastic integral to find the explicit solutions. We consider the Liouville form of the $\mathrm{ABm}$ with parameter $H \in\left(\frac{1}{2}, 1\right)$ to study the existence and uniqueness of strong solutions. Moreover, we consider an application of the equations in financial models.

(c) The Author(s) 2021. This article is licensed under a Creative Commons Attribution 4.0 International License, which permits use sharing, adaptation, distribution and reproduction in any medium or format, as long as you give appropriate credit to the original author(s) and the source, provide a link to the Creative Commons licence, and indicate if changes were made. The images or other third party material in this article are included in the article's Creative Commons licence, unless indicated otherwise in a credit line to the material. If material is not included in the article's Creative Commons licence and your intended use is not permitted by statutory regulation or exceeds the permitted use, you will need to obtain permission directly from the copyright holder. To view a copy of this licence, visit http://creativecommons.org/licenses/by/4.0/. 
The organization of the paper is as follows. In Sect. 2, the definition of the fBm and the Liouville form of this process are reviewed. Then some preliminaries on fuzzy stochastic processes and fuzzy stochastic integrals are recalled. In Sect. 3, a class of FSDEs driven by $\mathrm{fBm}$ is introduced. Besides, an approximation approach is used to prove the existence and uniqueness of the solutions. Finally, some conclusions are given in Sect. 4.

\section{Preliminaries}

\subsection{Fractional Brownian motion}

The $\mathrm{fBm} B^{H}=\left\{B^{H}(t), t \in[0, T]\right\}$ with Hurst parameter $H \in(0,1)$ is a zero mean Gaussian process with the following covariance function:

$$
R_{B^{H}}(t, s)=\mathbb{E}\left(B^{H}(t) B^{H}(s)\right)=\frac{1}{2}\left(s^{2 H}+t^{2 H}-|t-s|^{2 H}\right) .
$$

This process was introduced in [10] and studied in [16], where a stochastic integral representation was established in terms of the Brownian motion. The long-range dependence and self-similarity properties of this process, for $H>1 / 2$, yield a suitable driving noise in stochastic models, such as networks, finance, and physics. The classical Itô theory cannot be used to construct a stochastic integral in terms of the $\mathrm{fBm}$, because $B^{H}$ is not a semimartingale if $H \neq 1 / 2$. Two approaches have been used to define stochastic integrals with respect to $\mathrm{fBm}$. In the first one, the Riemann-Stieltjes stochastic integral can be defined using Young's integral [19] in the case of $H>1 / 2$. The second approach to defining a stochastic integral with respect to the $\mathrm{fBm}$ is based on the Malliavin calculus (see [2-4, 6]). In [16] a representation of $B_{H}(t)$ was given as follows:

$$
B^{H}(t)=\frac{1}{\Gamma(1+\alpha)}\left(\int_{-\infty}^{0}\left[(t-s)^{\alpha}-(-s)^{\alpha}\right] d W(s)+B_{H}(t)\right),
$$

where $W$ is a Brownian motion, $\alpha=H-\frac{1}{2}$ and $B_{H}(t)=\int_{0}^{t}(t-s)^{\alpha} d W(s)$. The process $B_{H}(t)$, with $H \in(0,1)$ is called the Liouville form of a fractional Brownian motion (LfBm) which holds many properties of the fBm except that it has non-stationary of increments. In [2] the Malliavin calculus technique was used to approximate $B_{H}(t)$ by a semimartingale process as follows:

$$
B_{H, \epsilon}(t)=\int_{0}^{t}(t-s+\epsilon)^{\alpha} d W(s), \quad \epsilon>0 .
$$

Furthermore,

$$
B_{H, \epsilon}(t)=\alpha \int_{0}^{t} \varphi^{\epsilon}(s) d s+\epsilon^{\alpha} W(t)
$$

where

$$
\varphi^{\epsilon}(t)=\int_{0}^{t}(t-s+\epsilon)^{\alpha-1} d W(s)
$$

The process $B_{H, \epsilon}(t)$ converges to $B_{H}(t)$ in $L^{2}(\Omega)$ when $\epsilon$ tends to zero [18]. 


\subsection{Fuzzy background}

In this section, we provide some preliminaries on fuzzy random variable, fuzzy stochastic process and fuzzy stochastic integral (see $[7,11,13])$. Let us denote by $\mathcal{K}(\mathbb{R})$ the family of all nonempty, compact and convex subsets of $\mathbb{R}$. The Hausdorff metric, denoted by $d_{H}$, is defined by

$$
d_{H}(A, B)=\max \left\{\sup _{a \in A} \inf _{b \in B}|a-b|, \sup _{b \in B} \inf _{a \in A}|a-b|\right\} .
$$

The space $\mathcal{K}(\mathbb{R})$ is a complete and separable metric space with respect to $d_{H}$. If $A, B, C \in$ $\mathcal{K}(\mathbb{R})$, then

$$
d_{H}(A+C, B+C)=d_{H}(A, B) .
$$

Let $(\Omega, \mathcal{A}, P)$ be a probability space. The mapping $F: \Omega \rightarrow \mathcal{K}(\mathbb{R})$ is called $\mathcal{A}$-measurable if it satisfies

$$
\{\omega \in \Omega: F(\omega) \cap C \neq \phi\} \in \mathcal{A},
$$

for every closed set $C \subset \mathbb{R}$. Let $\mathcal{M}(\Omega, \mathcal{A} ; \mathcal{K}(\mathbb{R}))$ denote a family of $\mathcal{A}$-measurable multifunctions with values in $\mathcal{K}(\mathbb{R})$. A multifunction $F \in \mathcal{M}$ is said to be $L^{p}$-integrably bounded, for $p \geq 1$, if there exists $h \in L^{p}\left(\Omega, \mathcal{A}, P ; \mathbb{R}_{+}\right)$such that $\|F\| \leq h$ P-a.e, $\mathbb{R}_{+}=[0, \infty)$, and

$$
\|F\|=d_{H}(F,\{0\})=\sup _{f \in F}|f| .
$$

It is well known that (see [8]) $F \in \mathcal{M}$ is $L^{p}$-integrably bounded if and only if $\|F\| \in$ $L^{p}\left(\Omega, \mathcal{A}, P ; \mathbb{R}_{+}\right)$. Let us denote

$$
\mathcal{L}^{p}(\Omega, \mathcal{A}, P ; \mathcal{K}(\mathbb{R}))=\left\{F \in \mathcal{M}(\Omega, \mathcal{A} ; \mathcal{K}(\mathbb{R})):\|F\| \in L^{p}\left(\Omega, \mathcal{A}, P ; \mathbb{R}_{+}\right)\right\} .
$$

The membership function $u: \mathbb{R} \rightarrow[0,1]$ is defined for a fuzzy set $u \in \mathbb{R}$, where $u(x)$ is the degree of membership of $x$ in the fuzzy set $u$. Let us denote by $\mathcal{F}(\mathbb{R})$ the fuzzy sets $u: \mathbb{R} \rightarrow[0,1]$ such that $[u]^{\alpha} \in \mathcal{K}(\mathbb{R})$ for every $\alpha \in[0,1]$, where $[u]^{\alpha}=\{x \in \mathbb{R}: u(x) \geq \alpha\}$.

Define $d_{\infty}: \mathcal{F}(\mathbb{R}) \times \mathcal{F}(\mathbb{R}) \rightarrow[0, \infty)$ by

$$
d_{\infty}(u, v)=\sup _{\alpha \in[0,1]} d_{H}\left([u]^{\alpha},[v]^{\alpha}\right)
$$

then $d_{\infty}$ is a metric in $\mathcal{F}(\mathbb{R})$ and $\left(\mathcal{F}(\mathbb{R}), d_{\infty}\right)$ is a complete metric space. For every $u, v, w, z \in$ $\mathcal{F}(\mathbb{R}), \lambda \in \mathbb{R}$, we have the following properties:

- $d_{\infty}(u+w, v+w)=d_{\infty}(u, v)$

- $d_{\infty}(u+v, w+z)=d_{\infty}(u, w)+d_{\infty}(v, z)$

- $d_{\infty}(u, v) \leq d_{\infty}(u, w)+d_{\infty}(w, v)$

- $d_{\infty}(\lambda u, \lambda v)=|\lambda| d_{\infty}(u, v)$.

We use $\langle 0\rangle \in \mathcal{F}(\mathbb{R})$ as $\langle 0\rangle:=\mathbb{1}_{\{0\}}$, where for $y \in \mathbb{R}, \mathbb{1}_{\{y\}}(x)=1$ if $x=y$ and $\mathbb{1}_{\{y\}}(x)=0$ if $x \neq y$.

Definition 2.1 ([17]) Let $(\Omega, \mathcal{A}, P)$ be a probability space. A fuzzy random variable is a function $X: \Omega \rightarrow \mathcal{F}(\mathbb{R})$, if the mapping $[X]^{\alpha}: \Omega \rightarrow \mathcal{K}(\mathbb{R})$ is an $\mathcal{A}$-measurable multifunction for all $\alpha \in[0,1]$. 
Let us consider a metric $\rho$ in the set $\mathcal{F}(\mathbb{R})$, and $\sigma$-algebra $\mathcal{B}_{\rho}$ generated by the topology induced by $\rho$. A fuzzy random variable can be viewed as a measurable mapping between two measurable spaces, namely $(\Omega, \mathcal{A})$ and $\left(\mathcal{F}(\mathbb{R}), \mathcal{B}_{\rho}\right)$, we call $X$ is $\mathcal{A} \mid \mathcal{B}_{\rho}$-measurable. Consider the following metric:

$$
d_{s}(u, v):=\inf _{\lambda \in \Lambda} \max \left\{\sup _{t \in[0,1]}|\lambda(t)-t|, \sup _{t \in[0,1]} d_{H}\left(\mathcal{X}_{u}(t), \mathcal{X}_{u}(\lambda(t))\right)\right\},
$$

where $\Lambda$ denotes the set of strictly increasing continuous functions $\lambda:[0,1] \rightarrow[0,1]$ such that $\lambda(0)=0, \lambda(1)=1$, and $\mathcal{X}_{u}, \mathcal{X}_{v}:[0,1] \rightarrow \mathcal{F}(\mathbb{R})$ are càdlàg representations for the fuzzy sets $u, v \in \mathcal{F}(\mathbb{R})$ (see [5]). The space $\left(\mathcal{F}(\mathbb{R}), d_{\infty}\right)$ is complete and non-separable, and the space $\left(\mathcal{F}(\mathbb{R}), d_{s}\right)$ is a Polish metric space.

For a mapping $X: \Omega \rightarrow \mathcal{F}(\mathbb{R})$ on the probability space $(\Omega, \mathcal{A}, P)$, we have:

- $X$ is a fuzzy random variable if and only if $X$ is $\mathcal{A} \mid \mathcal{B}_{d_{s}}$-measurable.

- If $X$ is $\mathcal{A} \mid \mathcal{B}_{d_{\infty}}$-measurable, then it is a fuzzy random variable; the opposite is not true.

Definition 2.2 A fuzzy random variable $X: \Omega \rightarrow \mathcal{F}(\mathbb{R})$, is $L^{p}$-integrably bounded, for $p \geq 1$, if $[X]^{\alpha} \in \mathcal{L}^{p}(\Omega, \mathcal{A}, P ; \mathcal{K}(\mathbb{R}))$, for every $\alpha \in[0,1]$.

Let us denote by $\mathcal{L}^{p}(\Omega, \mathcal{A}, P ; \mathcal{F}(\mathbb{R}))$ the set of all $L^{p}$-integrably bounded fuzzy random variables. The random variables $X, Y \in \mathcal{L}^{p}(\Omega, \mathcal{A}, P ; \mathcal{F}(\mathbb{R}))$ are identical if $P\left(d_{\infty}(X, Y)=0\right)=$ 1. For a fuzzy random variable $X: \Omega \rightarrow \mathcal{F}(\mathbb{R})$, and $p \geq 1$, the following conditions are equivalent:

a) $X \in \mathcal{L}^{p}(\Omega, \mathcal{A}, P ; \mathcal{F}(\mathbb{R}))$,

b) $[X]^{0} \in \mathcal{L}^{p}(\Omega, \mathcal{A}, P ; \mathcal{K}(\mathbb{R}))$,

c) $\left\|[X]^{0}\right\| \in L^{p}\left(\Omega, \mathcal{A}, P ; \mathbb{R}_{+}\right)$.

Let $I:=[0, T]$, and $(\Omega, \mathcal{A}, P)$ be a complete probability space with a filtration $\left\{\mathcal{A}_{t}\right\}_{t \in I}$ satisfying the hypotheses, an increasing and right continuous family of sub $\sigma$-algebras of $\mathcal{A}$, and containing all $P$-null sets.

Definition 2.3 If the mapping $X(t): \Omega \rightarrow \mathcal{F}(\mathbb{R})$, for every $t \in I$, is a fuzzy random variable, then $X: I \times \Omega \rightarrow \mathcal{F}(\mathbb{R})$ is a fuzzy stochastic process.

Definition 2.4 A fuzzy stochastic process $X$ is $d_{\infty}$-continuous, if almost all its trajectories, i.e. the mappings $X(\cdot, \omega): I \times \Omega \rightarrow \mathcal{F}(\mathbb{R})$ are $d_{\infty}$-continuous functions.

A fuzzy stochastic process $X$ is a measurable, if $[X]^{\alpha}: I \times \Omega \rightarrow \mathcal{K}(\mathbb{R})$ is $\mathcal{B}(I) \otimes \mathcal{A}$ measurable multifunction for all $\alpha \in[0,1]$, where $\mathcal{B}(I)$ denotes the Borel $\sigma$-algebra of subsets of $I$.

A process $X$ is nonanticipating if and only if for every $\alpha \in[0,1]$, the multifunction $[X]^{\alpha}$ is measurable with respect to the $\sigma$-algebra $\mathcal{N}$, which is defined as follows

$$
\mathcal{N}:=\left\{A \in \mathcal{B}(I) \otimes \mathcal{A}: A^{t} \in \mathcal{A}_{t} \text { for every } t \in I\right\},
$$

where $A^{t}=\{\omega:(t, \omega) \in A\}$. 
Definition 2.5 A fuzzy stochastic process $X$ is called $L^{p}$-integrably bounded ( $\left.p \geq 1\right)$, if there exists a real-valued stochastic process $h \in L^{p}\left(I \times \Omega, \mathcal{N} ; \mathbb{R}_{+}\right)$such that

$$
\left\|[X(t, \omega)]^{0}\right\| \mid \leq h(t, \omega),
$$

for almost all $(t, \omega) \in I \times \Omega$.

Let us denote by $\mathcal{L}^{p}(I \times \Omega, \mathcal{N} ; \mathcal{F}(\mathbb{R}))$ the set of nonanticipating and $L^{p}$-integrably bounded fuzzy stochastic processes. Let $X \in \mathcal{L}^{p}(I \times \Omega, \mathcal{N} ; \mathcal{F}(\mathbb{R}))$, by the Fubini theorem, the fuzzy integral is defined by

$$
\int_{0}^{T} X(s, \omega) d s
$$

for $\omega \in \Omega \backslash N_{x}$, where $N_{x} \in \mathcal{A}$ and $P\left(N_{x}\right)=0$. The fuzzy integral $\int_{0}^{T} X(s, \omega) d s$ can be defined level-wise. For every $\alpha \in[0,1]$, and every $\omega \in \Omega \backslash N_{x}$, the Aumann integral $\int_{0}^{T}[X(s, \omega)]^{\alpha} d s$ belongs to $\mathcal{K}(\mathbb{R})$, so a fuzzy random variable $\int_{0}^{T} X(s, \omega) d s$ belongs to $\mathcal{F}(\mathbb{R})$ for every $\omega \in$ $\Omega \backslash N_{x}$.

Definition 2.6 The fuzzy stochastic Lebesgue-Aumann integral of $X \in \mathcal{L}^{1}(I \times \Omega, \mathcal{N} ; \mathcal{F}(\mathbb{R}))$ is defined as

$$
L_{x}(t, \omega)= \begin{cases}\int_{0}^{T} \mathbb{1}_{[0, t]}(s) X(s, \omega) d s & \text { for every } \omega \in \Omega \backslash N_{x}, \\ \langle 0\rangle & \text { for every } \omega \in N_{x} .\end{cases}
$$

Proposition 2.1 ([13]) For the integral $L_{x}$, one can show the following properties:

1) Let $p \geq 1$. If $X \in \mathcal{L}^{p}(I \times \Omega, \mathcal{N} ; \mathcal{F}(\mathbb{R}))$, then $L_{x}(\cdot, \cdot) \in \mathcal{L}^{p}(I \times \Omega, \mathcal{N} ; \mathcal{F}(\mathbb{R}))$.

2) Let $X \in \mathcal{L}^{1}(I \times \Omega, \mathcal{N} ; \mathcal{F}(\mathbb{R}))$, then $\left\{L_{x}(t)\right\}_{t \in I}$ is $d_{\infty}$-continuous.

3) Let $X, Y \in \mathcal{L}^{p}(I \times \Omega, \mathcal{N} ; \mathcal{F}(\mathbb{R}))$, for $p \geq 1$, then

$$
\sup _{u \in[0, t]} d_{\infty}^{p}\left(L_{t, x}(u), L_{t, y}(u)\right) \leq t^{p-1} \int_{0}^{t} d_{\infty}^{p}(X(s), Y(s)) d s, \quad \text { a.e. }
$$

Let us denote by $\langle\cdot\rangle: \mathbb{R} \rightarrow \mathcal{F}(\mathbb{R})$ an embedding of $\mathbb{R}$ into $\mathcal{F}(\mathbb{R})$ i.e. for $r \in \mathbb{R}$,

$$
\langle r\rangle(a)= \begin{cases}1 & \text { for } a=r, \\ 0 & \text { for } a \in \mathbb{R} \backslash\{r\} .\end{cases}
$$

If $X: \Omega \rightarrow \mathbb{R}$ is a random variable on the probability space $(\Omega, \mathcal{A}, P)$, then $\langle X\rangle: \Omega \rightarrow$ $\mathcal{F}(\mathbb{R})$ is a fuzzy random variable. For stochastic processes we have a similar property.

We define the fuzzy stochastic Itô integral by using the fuzzy random variable as $\left\langle\int_{0}^{T} X(s) d W(s)\right\rangle$, where $W$ is a Wiener process. The following properties will be useful [13].

Proposition 2.2 Let $X \in L^{2}(I \times \Omega, \mathcal{N} ; \mathbb{R})$, then $\left\{\left\langle\int_{0}^{t} X(s) d W(s)\right\rangle\right\}_{t \in I}$ is a fuzzy stochastic process and we have $\left\langle\int_{0}^{t} X(s) d W(s)\right\rangle \in \mathcal{L}^{2}(I \times \Omega, \mathcal{N} ; \mathcal{F}(\mathbb{R}))$.

Proposition 2.3 Let $X \in L^{2}(I \times \Omega, \mathcal{N} ; \mathbb{R})$, then $\left\{\left\langle\int_{0}^{t} X(s) d W(s)\right\rangle\right\}_{t \in I}$ is $d_{\infty}$-continuous. 


\section{Application to fuzzy stochastic differential equation}

In this section we consider a class of FSDEs driven by the fBm which is given as follows:

$$
X(t)=X_{0}+\int_{0}^{t} f(s, X(s)) d s+\left\langle\int_{0}^{t} g(s, X(s)) d B_{H}(s)\right\rangle, \quad X_{0}=X(0),
$$

where $B_{H}$ is a Liouville form fBm with $H \in\left(\frac{1}{2}, 1\right), X_{0}: \Omega \rightarrow \mathcal{F}(\mathbb{R})$ is an FRV, $f: I \times \Omega \times$ $\mathcal{F}(\mathbb{R}) \rightarrow \mathcal{F}(\mathbb{R})$, and $g: I \times \Omega \times \mathcal{F}(\mathbb{R}) \rightarrow \mathbb{R}$. The corresponding approximation equation (3.1) is

$$
X^{\epsilon}(t)=X_{0}+\int_{0}^{t} f\left(s, X^{\epsilon}(s)\right) d s+\left\langle\int_{0}^{t} g\left(s, X^{\epsilon}(s)\right) d B_{H}^{\epsilon}(s)\right\rangle .
$$

Assumptions 3.1 Consider the following assumptions on the equation coefficients:

A1) The mappings $f: I \times \Omega \times \mathcal{F}(\mathbb{R}) \rightarrow \mathcal{F}(\mathbb{R})$ and $g: I \times \Omega \times \mathcal{F}(\mathbb{R}) \rightarrow \mathbb{R}$ are $\mathcal{N} \otimes \mathcal{B}_{d_{s}} \mid \mathcal{B}_{d_{s}}$-measurable and $\mathcal{N} \otimes \mathcal{B}_{d_{s}} \mid \mathcal{B}(\mathbb{R})$-measurable, respectively.

A2) For every $u, v \in \mathcal{F}(\mathbb{R})$ and every $t \in I$, there exists a constant $L>0$ such that

$$
\max \left\{d_{\infty}(f(t, \omega, u), f(t, \omega, v)),|g(t, \omega, u)-g(t, \omega, v)|\right\} \leq L d_{\infty}(u, v) .
$$

A3) For every $u, v \in \mathcal{F}(\mathbb{R})$ and every $t \in I$, there exists a constant $C>0$ such that

$$
\max \left\{d_{\infty}(f(t, \omega, u),\langle 0\rangle),|g(t, \omega, u)|\right\} \leq C\left(1+d_{\infty}(u,\langle 0\rangle)\right) .
$$

Proposition 3.1 ([13]) Consider $X, Y \in L^{2}(I \times \Omega, \mathcal{N} ; \mathbb{R})$, then

$$
\mathbb{E} \sup _{u \in[0, t]} d_{\infty}^{2}\left(\left\langle\int_{0}^{u} X(s) d W(s)\right\rangle,\left\langle\int_{0}^{u} Y(s) d W(s)\right\rangle\right) \leq 4 \mathbb{E} \int_{0}^{t} d_{\infty}^{2}(\langle X(s)\rangle,\langle Y(s)\rangle) d s,
$$

for every $t \in I$.

Theorem 3.2 Suppose that $: I \times \Omega \times \mathcal{F}(\mathbb{R}) \rightarrow \mathcal{F}(\mathbb{R})$ and $g: I \times \Omega \times \mathcal{F}(\mathbb{R}) \rightarrow \mathbb{R}$ as mappings satisfy assumptions $(A 1)-(A 3)$ and $X_{0} \in \mathcal{L}^{2}\left(\Omega, \mathcal{A}_{0}, P ; \mathcal{F}(\mathbb{R})\right)$. Then Eq. (3.2) has a strong unique solution.

Proof Consider the SDE (3.2),

$$
X^{\epsilon}(t)=X_{0}+\int_{0}^{t} f\left(s, X^{\epsilon}(s)\right) d s+\left\langle\int_{0}^{t} g\left(s, X^{\epsilon}(s)\right) d B_{H}^{\epsilon}(s)\right\rangle .
$$

By Eq. (2.3), we can write

$$
\begin{aligned}
X^{\epsilon}(t)= & X_{0}+\int_{0}^{t} f\left(s, X^{\epsilon}(s)\right) d s \\
& +\left\langle\int_{0}^{t} \alpha \varphi^{\epsilon}(s) g\left(s, X^{\epsilon}(s)\right) d s+\int_{0}^{t} \epsilon^{\alpha} g\left(s, X^{\epsilon}(s)\right) d W(s)\right\rangle .
\end{aligned}
$$

Let us consider the Picard iterations

$$
X_{n}^{\epsilon}(t)=X_{0}+\int_{0}^{t} f\left(s, X_{n-1}^{\epsilon}(s)\right) d s
$$




$$
+\left\langle\int_{0}^{t} \alpha \varphi^{\epsilon}(s) g\left(s, X_{n-1}^{\epsilon}(s)\right) d s+\int_{0}^{t} \epsilon^{\alpha} g\left(s, X_{n-1}^{\epsilon}(s)\right) d W(s)\right\rangle, \quad \text { a.e., }
$$

for $n=1,2, \ldots$, and for every $t \in I$, and $X_{0}(t)=X_{0}$. For $t \in I$ and $n \in \mathbb{N}$ we denote

$$
j_{n}(t)=\mathbb{E} \sup _{u \in[0, t]} d_{\infty}^{2}\left(X_{n}^{\epsilon}(u), X_{n-1}^{\epsilon}(u)\right)
$$

Then, by Propositions 2.1, 3.1, and assumption A3, it can be written as

$$
\begin{aligned}
j_{1}(t)= & \mathbb{E} \sup _{u \in[0, t]} d_{\infty}^{2}\left(\int_{0}^{u} f\left(s, X_{0}^{\epsilon}(s)\right) d s\right. \\
& \left.+\left\langle\int_{0}^{u} \alpha \varphi^{\epsilon}(s) g\left(s, X_{0}^{\epsilon}(s)\right) d s+\int_{0}^{u} \epsilon^{\alpha} g\left(s, X_{0}^{\epsilon}(s)\right) d W(s)\right\rangle,\langle 0\rangle\right) \\
\leq & 3 \mathbb{E} \sup _{u \in[0, t]} d_{\infty}^{2}\left(\int_{0}^{u} f\left(s, X_{0}^{\epsilon}(s)\right) d s,\langle 0\rangle\right) \\
& +3 \mathbb{E} \sup _{u \in[0, t]} d_{\infty}^{2}\left(\left\langle\int_{0}^{u} \alpha \varphi^{\epsilon}(s) g\left(s, X_{0}^{\epsilon}(s)\right) d s\right\rangle,\langle 0\rangle\right) \\
& +3 \mathbb{E} \sup _{u \in[0, t]} d_{\infty}^{2}\left(\left\langle\int_{0}^{u} \epsilon^{\alpha} g\left(s, X_{0}^{\epsilon}(s)\right) d W(s)\right\rangle,\langle 0\rangle\right) \\
\leq & 3 t \mathbb{E} \int_{0}^{t} d_{\infty}^{2}\left(f\left(s, X_{0}^{\epsilon}(s)\right),\langle 0\rangle\right) d s+3 \alpha^{2} \mathbb{E} \sup _{u \in[0, t]} d_{\infty}^{2}\left(\left\langle\int_{0}^{u} \varphi^{\epsilon}(s) g\left(s, X_{0}^{\epsilon}(s)\right) d s\right\rangle,\langle 0\rangle\right) \\
& +12 \epsilon^{2 \alpha} \mathbb{E} \int_{0}^{t} d_{\infty}^{2}\left(\left\langle g\left(s, X_{0}^{\epsilon}(s)\right)\right\rangle,\langle 0\rangle\right) d s \\
\leq & 6 C^{2}\left(T+4 \epsilon^{2 \alpha}\right)\left(1+\mathbb{E}\left|\left\|\left[X^{\epsilon}\right]^{0} \mid\right\|^{2}\right) t\right. \\
& +3 \alpha^{2} \mathbb{E} \sup _{u \in[0, t]} d_{\infty}^{2}\left(\left\langle\int_{0}^{u} \varphi^{\epsilon}(s) g\left(s, X_{0}^{\epsilon}(s)\right) d s\right\rangle,\langle 0\rangle\right),
\end{aligned}
$$

for $\alpha=H-\frac{1}{2}>0$. Hence

$$
\begin{aligned}
j_{1}(t) \leq & 6 C^{2}\left(T+4 \epsilon^{2 \alpha}\right)\left(1+\mathbb{E}\left\|\left[X^{\epsilon}\right]^{0}\right\|^{2}\right) t \\
& +3 \alpha^{2} \mathbb{E} \sup _{u \in[0, t]} d_{\infty}^{2}\left(\left\langle\int_{0}^{u} \varphi^{\epsilon}(s) g\left(s, X_{0}^{\epsilon}(s)\right) d s\right\rangle,\langle 0\rangle\right) .
\end{aligned}
$$

We have

$$
\begin{aligned}
& \mathbb{E} \sup _{u \in[0, t]} d_{\infty}^{2}\left(\left\langle\int_{0}^{u} \varphi^{\epsilon}(s) g\left(s, X_{0}^{\epsilon}(s)\right) d s\right\rangle,\langle 0\rangle\right) \\
& \leq \mathbb{E} \sup _{u \in[0, t]} d_{H}^{2}\left(\left\{\int_{0}^{u} \varphi^{\epsilon}(s) g\left(s, X_{0}^{\epsilon}(s)\right) d s\right\},\{0\}\right) \\
& \leq \mathbb{E} \sup _{u \in[0, t]}\left(\int_{0}^{u} \varphi^{\epsilon}(s) g\left(s, X_{0}^{\epsilon}(s)\right) d s\right)^{2} .
\end{aligned}
$$

By applying (2.4) to (3.8), and the Hölder inequality we get

$$
\mathbb{E} \sup _{u \in[0, t]}\left(\int_{0}^{u} \varphi^{\epsilon}(s) g\left(s, X_{0}^{\epsilon}(s)\right) d s\right)^{2}
$$




$$
\begin{aligned}
& =\mathbb{E} \sup _{u \in[0, t]}\left(\int_{0}^{u}\left(\int_{0}^{s}(s-r+\epsilon)^{\alpha-1} d W(r)\right) g\left(s, X_{0}^{\epsilon}(s)\right) d s\right)^{2} \\
& =\mathbb{E} \sup _{u \in[0, t]}\left(\int_{0}^{u} \int_{s}^{u} g\left(r, X_{0}^{\epsilon}(r)\right)(r-s+\epsilon)^{\alpha-1} d r d W(s)\right)^{2} \\
& \leq 4 \mathbb{E} \int_{0}^{t}\left(\int_{s}^{t} g\left(r, X_{0}^{\epsilon}(r)\right)(r-s+\epsilon)^{\alpha-1} d r\right)^{2} d s \\
& \leq 4 \mathbb{E} \int_{0}^{t}\left(\int_{s}^{t} g^{2}\left(r, X_{0}^{\epsilon}(r)\right)(r-s+\epsilon)^{\alpha-1} d r\right)\left(\int_{s}^{t}(r-s+\epsilon)^{\alpha-1} d r\right) d s \\
& \leq \frac{4}{\alpha^{2}}(t+\epsilon)^{\alpha} \mathbb{E} \int_{0}^{t} g^{2}\left(r, X_{0}^{\epsilon}(r)\right)(r+\epsilon)^{\alpha} d r \\
& \leq \frac{4}{\alpha^{2}}(t+\epsilon)^{2 \alpha} \mathbb{E} \int_{0}^{t} g^{2}\left(r, X_{0}^{\epsilon}(r)\right) d r \\
& \leq \frac{8 C^{2}}{\alpha^{2}}(T+\epsilon)^{2 \alpha}\left(1+\mathbb{E}\left\|\left[X_{0}^{\epsilon}\right]^{0}\right\| \|^{2}\right) t .
\end{aligned}
$$

Hence, from (3.7) and (3.9) we obtain

$$
j_{1}(t) \leq 6 C^{2}\left(T+4 \epsilon^{2 \alpha}+4(T+\epsilon)^{2 \alpha}\right)\left(1+\mathbb{E}\left\|\left[X_{0}^{\epsilon}\right]^{0}\right\|^{2}\right) t,
$$

for every $t \in I$. Then, similarly,

$$
\begin{aligned}
j_{n+1}(t) & \leq 3\left(t+4 \epsilon^{2 \alpha}+4(t+\epsilon)^{2 \alpha}\right) L^{2} \mathbb{E} \int_{0}^{t} d_{\infty}^{2}\left(X_{n}^{\epsilon}(u), X_{n-1}^{\epsilon}(u)\right) d s \\
& \leq 3\left(t+4 \epsilon^{2 \alpha}+4(t+\epsilon)^{2 \alpha}\right) L^{2} \int_{0}^{t} \mathbb{E} \sup _{u \in[0, s]} d_{\infty}^{2}\left(X_{n}^{\epsilon}(u), X_{n-1}^{\epsilon}(u)\right) d s \\
& \leq 3\left(t+4 \epsilon^{2 \alpha}+4(t+\epsilon)^{2 \alpha}\right) L^{2} \int_{0}^{t} j_{n}(s) d s
\end{aligned}
$$

Therefore

$$
j_{n}(t) \leq 2 C^{2} 3^{n}\left(t+4 \epsilon^{2 \alpha}+4(t+\epsilon)^{2 \alpha}\right)^{n}\left(1+\left.\mathbb{E}\left\|\left[X_{0}^{\epsilon}\right]^{0}\right\|\right|^{2}\right) L^{2(n-1)} \frac{t^{n}}{n !}, \quad t \in I, n \in \mathbb{N}
$$

Apply the Chebyshev inequality, it follows that

$$
P\left(\sup _{u \in I} d_{\infty}^{2}\left(X_{n}^{\epsilon}(u), X_{n-1}^{\epsilon}(u)\right)>\frac{1}{2^{n}}\right) \leq 2^{n} j_{n}(T)
$$

The series $\sum_{n=1}^{\infty} 2^{n} j_{n}(T)$ is convergent. From the Borel-Cantelli lemma, we derive

$$
P\left(\sup _{u \in I} d_{\infty}^{2}\left(X_{n}^{\epsilon}(u), X_{n-1}^{\epsilon}(u)\right)>\frac{1}{(\sqrt{2})^{n}} \text { infinitely often }\right)=0
$$

For almost all $\omega \in \Omega$, there exists $n_{0}(\omega)$ such that

$$
\sup _{u \in I} d_{\infty}^{2}\left(X_{n}^{\epsilon}(u), X_{n-1}^{\epsilon}(u)\right) \leq \frac{1}{(\sqrt{2})^{n}}, \quad \text { if } n \geq n_{0}
$$


The sequence $\left\{X_{n}^{\epsilon}(\cdot, \omega)\right\}$ is uniformly convergent to a $d_{\infty}^{2}$-continuous fuzzy process $\widetilde{X}^{\epsilon}(\cdot, \omega)$ for every $\omega \in \Omega_{c}$, in which $\Omega_{c} \in \mathcal{A}$ and $P\left(\Omega_{c}\right)=1$. We can define the mapping $X^{\epsilon}: I \times \Omega \rightarrow$ $\mathcal{F}(\mathbb{R})$, as $X^{\epsilon}(\cdot, \omega)=\widetilde{X}^{\epsilon}(\cdot, \omega)$ if $\omega \in \Omega_{c}$ and $X^{\epsilon}(\cdot, \omega)$ as freely chosen fuzzy function when $\omega \in \Omega \backslash \Omega_{c}$. For every $\alpha \in[0,1]$ and every $t \in I$ with a.e., we have

$$
d_{H}\left(\left[X_{n}^{\epsilon}(t)\right]^{\alpha},\left[X^{\epsilon}(t)\right]^{\alpha}\right) \rightarrow 0 \quad \text { as } n \rightarrow \infty .
$$

Hence, $X^{\epsilon}$ will be a continuous fuzzy stochastic process. Then, by $X_{n}^{\epsilon} \in \mathcal{L}^{2}(I \times \Omega, \mathcal{N} ; \mathcal{F}(\mathbb{R}))$, we get $X^{\epsilon} \in \mathcal{L}^{2}(I \times \Omega, \mathcal{N} ; \mathcal{F}(\mathbb{R}))$. Hence, as $n$ goes to infinity, we can verify that

$$
\mathbb{E} \sup _{t \in I}\left[d_{\infty}^{2}\left(X_{n}^{\epsilon}(t), X^{\epsilon}(t)\right)+d_{\infty}^{2}\left(X_{n}^{\epsilon}(t), X_{0}^{\epsilon}+\int_{0}^{t} f\left(s, X^{\epsilon}(s)\right) d s+\left\langle\int_{0}^{t} g\left(s, X^{\epsilon}(s)\right) d B_{H}^{\epsilon}(s)\right\rangle\right)\right]^{2}
$$

tends to zero. Then

$$
\mathbb{E} \sup _{t \in I} d_{\infty}^{2}\left[\left(X^{\epsilon}(t), X_{0}^{\epsilon}+\int_{0}^{t} f\left(s, X^{\epsilon}(s)\right) d s+\left\langle\int_{0}^{t} g\left(s, X^{\epsilon}(s)\right) d B_{H}^{\epsilon}(s)\right\rangle\right)\right]=0 .
$$

Therefore

$$
\sup _{t \in I} d_{\infty}^{2}\left[\left(X^{\epsilon}(t), X_{0}^{\epsilon}+\int_{0}^{t} f\left(s, X^{\epsilon}(s)\right) d s+\left\langle\int_{0}^{t} g\left(s, X^{\epsilon}(s)\right) d B_{H}^{\epsilon}(s)\right\rangle\right)\right]=0,
$$

which shows the existence of the strong solution.

Now, $X^{\epsilon}, Y^{\epsilon}: I \times \Omega \rightarrow \mathcal{F}(\mathbb{R})$ are assumed to be strong solutions. Consider

$$
j(t)=\mathbb{E} \sup _{u \in[0, t]} d_{\infty}^{2}\left(X^{\epsilon}(u), Y^{\epsilon}(u)\right)
$$

then, by computations similar to the existence case, we have

$$
j(t) \leq 3\left(t+4 \epsilon^{2 \alpha}+4(t+\epsilon)^{2 \alpha}\right) L^{2} \mathbb{E} \int_{0}^{t} d_{\infty}^{2}\left(X^{\epsilon}(s), Y^{\epsilon}(s)\right) d s \leq 3\left(T+4 \epsilon^{2 \alpha}+\alpha^{2}\right) L^{2} \int_{0}^{t} j(s) d s .
$$

The implementation of the Gronwall inequality leads to $j(t)=0$ for $t \in I$. Then

$$
\sup _{t \in I} d_{\infty}^{2}\left(X^{\epsilon}(t), Y^{\epsilon}(t)\right)=0, \quad \text { a.e., }
$$

by which the proof of uniqueness is completed.

Lemma 3.1 For every $\epsilon>0$ and $0<\alpha<\frac{1}{2}$ we have

$$
\int_{s}^{t}\left((r-s+\epsilon)^{\alpha-1}-(r-s)^{\alpha-1}\right) d r \leq \frac{\alpha+1}{\alpha} \epsilon^{\alpha}
$$

Proof We apply the finite-increments formula to the function $f(x)=x^{\alpha-1}$ to obtain

$$
(x+\epsilon)^{\alpha-1}-x^{\alpha-1}=(\alpha-1)(x+\theta \epsilon)^{(\alpha-2)} \epsilon, \quad 0<\theta<1,
$$


then

$$
\left|(r-s+\epsilon)^{\alpha-1}-(r-s)^{\alpha-1}\right| \leq|\alpha-1||r-s|^{\alpha-2} \epsilon .
$$

Hence

$$
\begin{aligned}
\int_{s}^{t}\left|(r-s+\epsilon)^{\alpha-1}-(r-s)^{\alpha-1}\right| d r= & \int_{s}^{s+\epsilon}\left|(r-s+\epsilon)^{\alpha-1}-(r-s)^{\alpha-1}\right| d r \\
& +\int_{s+\epsilon}^{t}\left|(r-s+\epsilon)^{\alpha-1}-(r-s)^{\alpha-1}\right| d r \\
\leq & \int_{s}^{s+\epsilon}\left|(2(r-s))^{\alpha-1}-(r-s)^{\alpha-1}\right| d r \\
& +|\alpha-1| \epsilon \int_{s+\epsilon}^{t}|r-s|^{\alpha-2} d r .
\end{aligned}
$$

Therefore

$$
\begin{aligned}
\int_{s}^{t}\left|(r-s+\epsilon)^{\alpha-1}-(r-s)^{\alpha-1}\right| d r & \leq \int_{s}^{s+\epsilon}(r-s)^{\alpha-1} d r+|\alpha-1| \epsilon \int_{s+\epsilon}^{t}(r-s)^{\alpha-2} d r \\
& \leq \frac{1}{\alpha} \epsilon^{\alpha}+|\alpha-1| \epsilon\left(\frac{1}{\alpha-1} \epsilon^{\alpha-1}\right) \\
& =\frac{\alpha+1}{\alpha} \epsilon^{\alpha} .
\end{aligned}
$$

Proposition 3.2 The solution $X^{\epsilon}(t)$ of Eq. (3.2) converges to the solution $X(t)$ of Eq. (3.1) in $\mathcal{L}^{2}(I \times \Omega)$ as $\epsilon \rightarrow 0$ uniformly with respect to $t \in[0, T]$.

Proof Consider Eq. (3.1) and the corresponding approximation of equations as follows:

$$
\begin{aligned}
& X(t)=X_{0}+\int_{0}^{t} f(s, X(s)) d s+\left\langle\int_{0}^{t} g(s, X(s)) d B_{H}(s)\right\rangle \\
& X^{\epsilon}(t)=X_{0}+\int_{0}^{t} f\left(s, X^{\epsilon}(s)\right) d s+\left\langle\int_{0}^{t} g\left(s, X^{\epsilon}(s)\right) d B_{H}^{\epsilon}(s)\right\rangle .
\end{aligned}
$$

We can write

$$
\begin{aligned}
\mathbb{E} \sup _{u \in[0, t]} d_{\infty}^{2}\left(X(u), X^{\epsilon}(u)\right) \\
\leq 2 \mathbb{E} \sup _{u \in[0, t]} \int_{0}^{u} d_{\infty}^{2}\left(f(s, X(s)), f\left(s, X^{\epsilon}(s)\right)\right) d s \\
\quad+2 \mathbb{E} \sup _{u \in[0, t]} d_{\infty}^{2}\left(\left\langle\int_{0}^{u} g(s, X(s)) d B_{H}(s)\right\rangle,\left\langle\int_{0}^{u} g\left(s, X^{\epsilon}(s)\right) d B_{H}^{\epsilon}(s)\right\rangle\right) .
\end{aligned}
$$

Then

$$
\mathbb{E} \sup _{u \in[0, t]} d_{\infty}^{2}\left(X(u), X^{\epsilon}(u)\right)
$$




$$
\begin{aligned}
& \leq 2 \mathbb{E} \sup _{u \in[0, t]} \int_{0}^{u} d_{\infty}^{2}\left(f(s, X(s)), f\left(s, X^{\epsilon}(s)\right)\right) d s \\
& +4 \mathbb{E} \sup _{u \in[0, t]} d_{\infty}^{2}\left(\left\langle\int_{0}^{u} g(s, X(s)) d B_{H}(s)\right\rangle,\left\langle\int_{0}^{u} g(s, X(s)) d B_{H}^{\epsilon}(s)\right\rangle\right) \\
& +4 \mathbb{E} \sup _{u \in[0, t]} d_{\infty}^{2}\left(\left\langle\int_{0}^{u} g(s, X(s)) d B_{H}^{\epsilon}(s)\right\rangle,\left\langle\int_{0}^{u} g\left(s, X^{\epsilon}(s)\right) d B_{H}^{\epsilon}(s)\right\rangle\right) \\
& =2 \mathbb{E} \int_{0}^{t} d_{\infty}^{2}\left(f(s, X(s)), f\left(s, X^{\epsilon}(s)\right)\right) d s \\
& +4 \mathbb{E} \sup _{u \in[0, t]}\left|\int_{0}^{u} g\left(s, X^{\epsilon}(s)\right)\left(d B_{H}^{\epsilon}(s)-d B_{H}(s)\right)\right|^{2} \\
& +4 \mathbb{E} \sup _{u \in[0, t]} \mid \int_{0}^{u}\left(g(s, X(s))-\left.g\left(s, X^{\epsilon}(s)\right) d B_{H}^{\epsilon}(s)\right|^{2} .\right.
\end{aligned}
$$

Apply Eq. (2.3) to get

$$
\begin{aligned}
& \mathbb{E} \sup _{u \in[0, t]} d_{\infty}^{2}\left(X(u), X^{\epsilon}(u)\right) \\
& \leq 2 \mathbb{E} \int_{0}^{t} d_{\infty}^{2}\left(f(s, X(s)), f\left(s, X^{\epsilon}(s)\right)\right) d s \\
&+ 8 \epsilon^{2 \alpha} \mathbb{E} \sup _{u \in[0, t]}\left|\int_{0}^{u} g\left(s, X^{\epsilon}(s)\right) d W(s)\right|^{2} \\
&+ 8 \alpha^{2} \mathbb{E} \sup _{u \in[0, t]}\left|\int_{0}^{u} g\left(s, X^{\epsilon}(s)\right)\left(\int_{0}^{s}\left((s-r+\epsilon)^{\alpha-1}-(s-r)^{\alpha-1}\right) d W(r)\right) d s\right|^{2} \\
&+8 \alpha^{2} \mathbb{E} \sup _{u \in[0, t]} \mid \int_{0}^{u}\left(g(s, X(s))-\left.g\left(s, X^{\epsilon}(s)\right)\left(\int_{0}^{s}(s-r+\epsilon)^{\alpha-1} d W(r)\right) d s\right|^{2}\right. \\
&+8 \epsilon^{2 \alpha} \mathbb{E} \sup _{u \in[0, t]} \mid \int_{0}^{u}\left(g(s, X(s))-\left.g\left(s, X^{\epsilon}(s)\right) d W(s)\right|^{2} .\right.
\end{aligned}
$$

Then

$$
\begin{aligned}
& \mathbb{E} \sup _{u \in[0, t]} d_{\infty}^{2}\left(X(u), X^{\epsilon}(u)\right) \\
& \leq 2 \mathbb{E} \int_{0}^{t} d_{\infty}^{2}\left(f(s, X(s)), f\left(s, X^{\epsilon}(s)\right)\right) d s \\
& +8 \epsilon^{2 \alpha} \mathbb{E} \sup _{u \in[0, t]}\left|\int_{0}^{u} g\left(s, X^{\epsilon}(s)\right) d W(s)\right|^{2} \\
& +8 \alpha^{2} \mathbb{E} \sup _{u \in[0, t]}\left|\int_{0}^{u} \int_{s}^{u} g\left(r, X^{\epsilon}(r)\right)\left((r-s+\epsilon)^{\alpha-1}-(r-s)^{\alpha-1}\right) d r d W(s)\right|^{2} \\
& +8 \alpha^{2} \mathbb{E} \sup _{u \in[0, t]} \mid \int_{0}^{u} \int_{s}^{u}\left(g(r, X(r))-\left.g\left(s, X^{\epsilon}(s)\right)(r-s+\epsilon)^{\alpha-1} d r d W(s)\right|^{2}\right. \\
& +8 \epsilon^{2 \alpha} \mathbb{E} \sup _{u \in[0, t]} \mid \int_{0}^{u}\left(g(s, X(s))-\left.g\left(s, X^{\epsilon}(s)\right) d W(s)\right|^{2} .\right.
\end{aligned}
$$


Apply the Doob inequality, the Hölder inequality and the Itô isometry property to obtain

$$
\begin{aligned}
\mathbb{E} \sup _{u \in[0, t]} d_{\infty}^{2}\left(X(u), X^{\epsilon}(u)\right) \\
\leq \quad 2 \mathbb{E} \int_{0}^{t} d_{\infty}^{2}\left(f(s, X(s)), f\left(s, X^{\epsilon}(s)\right)\right) d s+32 \epsilon^{2 \alpha} \mathbb{E} \int_{0}^{t} g^{2}\left(s, X^{\epsilon}(s)\right) d s \\
\quad+32 \alpha^{2} \mathbb{E} \int_{0}^{t}\left[\int_{s}^{t} g^{2}\left(r, X^{\epsilon}(r)\right)\left((r-s+\epsilon)^{\alpha-1}-(r-s)^{\alpha-1}\right) d r\right. \\
\left.\quad \times \int_{s}^{t}\left((r-s+\epsilon)^{\alpha-1}-(r-s)^{\alpha-1}\right) d r\right] d s \\
+32 \alpha^{2} \mathbb{E} \int_{0}^{t}\left(\int_{s}^{t}\left(g(r, X(r))-g\left(s, X^{\epsilon}(s)\right)^{2}(r-s+\epsilon)^{\alpha-1} d r\right)\right. \\
\quad \times\left(\int_{s}^{t}(r-s+\epsilon)^{\alpha-1} d r\right) d s \\
\quad+32 \epsilon^{2 \alpha} \mathbb{E} \int_{0}^{t}\left(g(s, X(s))-g\left(s, X^{\epsilon}(s)\right)^{2} d s .\right.
\end{aligned}
$$

By similar arguments to (3.9), from (3.11) and assumptions (A1)-(A3), we infer that

$$
\begin{aligned}
& \mathbb{E} \sup _{u \in[0, t]} d_{\infty}^{2}\left(X(u), X^{\epsilon}(u)\right) \\
& \leq 2 L^{2} \mathbb{E} \int_{0}^{t} d_{\infty}^{2}\left(X(s), X^{\epsilon}(s)\right) d s+64 \epsilon^{2 \alpha} C^{2} \int_{0}^{t}\left(1+\mathbb{E}\left\|\left[X^{\epsilon}(s)\right]^{0}\right\| \|^{2}\right) d s \\
&+64 C^{2}(T+\epsilon)^{2 \alpha} \frac{\alpha+1}{\alpha} \epsilon^{\alpha} \int_{0}^{t}\left(1+\mathbb{E}\left\|\left[X^{\epsilon}(s)\right]^{0}\right\|^{2}\right) d s \\
&+32 L^{2}(T+\epsilon)^{2 \alpha} \mathbb{E} \int_{0}^{t} d_{\infty}^{2}\left(X(s), X^{\epsilon}(s)\right) d s \\
&+32 L^{2} \epsilon^{2 \alpha} \mathbb{E} \int_{0}^{t} d_{\infty}^{2}\left(X(s), X^{\epsilon}(s)\right) d s .
\end{aligned}
$$

Hence

$$
\begin{aligned}
\mathbb{E} \sup _{u \in[0, t]} d_{\infty}^{2}\left(X(u), X^{\epsilon}(u)\right) \\
\leq\left(2 L^{2}+32 L^{2}(T+\epsilon)^{2 \alpha}+32 L^{2} \epsilon^{2 \alpha}\right) \int_{0}^{t} \mathbb{E} \sup _{u \in[0, s]} d_{\infty}^{2}\left(X(u), X^{\epsilon}(u)\right) d s \\
\quad+\left(64 \epsilon^{2 \alpha} C^{2}+64 C^{2}(T+\epsilon)^{2 \alpha} \frac{\alpha+1}{\alpha} \epsilon^{\alpha}\right) \int_{0}^{t}\left(1+\mathbb{E}\left\|\left[X^{\epsilon}(s)\right]^{0}\right\|^{2}\right) d s .
\end{aligned}
$$

By Gronwall's lemma, $\mathbb{E} \sup _{u \in[0, t]} d_{\infty}^{2}\left(X(u), X^{\epsilon}(u)\right) \rightarrow 0$ as $\epsilon \rightarrow 0$, which completes the proof.

\subsection{Example in finance}

The following crisp SFDE is typically used in financial modeling:

$$
X(t)=X_{0}+\int_{0}^{t} \mu X(s) d s+\int_{0}^{t} \sigma X(s) d B_{H}(s), \quad X_{0}=X(0),
$$


where the underlying stochastic process is the $\mathrm{fBm}$. The long-range dependence and selfsimilarity property of $\mathrm{fBm}$ make this process suitable to describe the financial quantities. On the other hand, we can model the price dynamics by the equation which involves the uncertainties. This leads to modeling with fuzzy processes in equations. In the case of linear coefficients, we obtain an explicit solution of Eq. (3.1). Thus, consider the fractional FSDE which satisfy assumptions of Theorem 3.2 as follows:

$$
X(t)=X_{0}+\int_{0}^{t} \mu X(s) d s+\left\langle\int_{0}^{t} \frac{\sigma}{2}\left(X_{l}^{1}(s)+X_{u}^{1}(s)\right) d B_{H}(s)\right\rangle
$$

where $X: \mathbb{R}_{+} \times \Omega \rightarrow \mathcal{F}(\mathbb{R}), B_{H}$ is an $\mathrm{fBm}, X_{l}^{1}, X_{u}^{1}: \mathbb{R}_{+} \times \Omega \rightarrow \mathbb{R}$ such that $[X(t)]^{1}=$ $\left[X_{l}^{1}(t), X_{u}^{1}(t)\right], X_{0} \in \mathcal{L}^{2}\left(\Omega, \mathcal{A}_{0}, P ; \mathcal{F}(\mathbb{R})\right)$, and $\mu, \sigma \in \mathbb{R}$. In order to find a closed explicit form of a solution to (3.24), for $\mu \geq 0$, we obtain the following system of equations:

$$
\left\{\begin{array}{l}
X_{l}^{1}(t)=X_{l}^{1}(0)+\int_{0}^{t} \mu X_{l}^{1}(s) d s+\int_{0}^{t} \frac{\sigma}{2}\left(X_{l}^{1}(s)+X_{u}^{1}(s)\right) d B_{H}(s), \\
X_{u}^{1}(t)=X_{u}^{1}(0)+\int_{0}^{t} \mu X_{u}^{1}(s) d s+\int_{0}^{t} \frac{\sigma}{2}\left(X_{l}^{1}(s)+X_{u}^{1}(s)\right) d B_{H}(s),
\end{array}\right.
$$

then

$$
\begin{aligned}
X_{l}^{1}(t)+X_{u}^{1}(t)= & X_{l}^{1}(0)+X_{u}^{1}(0)+\int_{0}^{t} \mu\left(X_{l}^{1}(s)+X_{u}^{1}(s)\right) d s \\
& +\int_{0}^{t} \sigma\left(X_{l}^{1}(s)+X_{u}^{1}(s)\right) d B_{H}(s) .
\end{aligned}
$$

By equation (2.3), the approximation form of Eq. (3.25) is

$$
\begin{aligned}
X_{l}^{\epsilon 1}(t)+X_{u}^{\epsilon 1}(t)= & X_{l}^{\epsilon 1}(0)+X_{u}^{\epsilon 1}(0)+\int_{0}^{t}\left(\mu+\sigma \alpha \varphi^{\epsilon}(s)\right)\left(X_{l}^{\epsilon 1}(s)+X_{u}^{\epsilon 1}(s)\right) d s \\
& +\int_{0}^{t} \sigma \epsilon^{\alpha}\left(X_{l}^{\epsilon 1}(s)+X_{u}^{\epsilon 1}(s)\right) d W(s) .
\end{aligned}
$$

Hence, from the explicit solution of the crisp linear SDEs, one has a unique solution,

$$
\begin{aligned}
X_{l}^{\epsilon 1}(t)+X_{u}^{\epsilon 1}(t) & =\left(X_{l}^{\epsilon 1}(0)+X_{u}^{\epsilon 1}(0)\right) \exp \left(\mu t+\sigma \alpha \int_{0}^{t} \varphi^{\epsilon}(s) d s-\frac{1}{2} \sigma^{2} \epsilon^{2 \alpha} t+\sigma \epsilon^{\alpha} W(t)\right) \\
& =\left(X_{l}^{\epsilon 1}(0)+X_{u}^{\epsilon 1}(0)\right) \exp \left(\mu t+\sigma B_{H}^{\epsilon}(t)-\frac{1}{2} \sigma^{2} \epsilon^{2 \alpha} t\right) .
\end{aligned}
$$

Now, for every $\alpha \in[0,1]$, we apply a similar procedure to obtain the following systems:

$$
\left\{\begin{array}{l}
X_{l}^{\epsilon \alpha}(t)=X_{l}^{\epsilon \alpha}(0)+\int_{0}^{t} \mu X_{l}^{\epsilon \alpha}(s) d s+\int_{0}^{t} \frac{\sigma}{2}\left(X_{l}^{\epsilon 1}(s)+X_{u}^{\epsilon 1}(s)\right) d B_{H}^{\epsilon}(s), \\
X_{u}^{\epsilon \alpha}(t)=X_{u}^{\epsilon \alpha}(0)+\int_{0}^{t} \mu X_{u}^{\epsilon \alpha}(s) d s+\int_{0}^{t} \frac{\sigma}{2}\left(X_{l}^{\epsilon 1}(s)+X_{u}^{\epsilon 1}(s)\right) d B_{H}^{\epsilon}(s) .
\end{array}\right.
$$

For $\mu \geq 0$, we apply the solution (3.27) to get the following system:

$$
X_{l}^{\epsilon \alpha}(t)=X_{l}^{\epsilon \alpha}(0)+\int_{0}^{t} \mu X_{l}^{\epsilon \alpha}(s) d s
$$




$$
\begin{aligned}
& +\int_{0}^{t} \frac{\sigma}{2}\left(X_{l}^{\epsilon 1}(0)+X_{u}^{\epsilon 1}(0)\right) \exp \left(\mu s+\sigma B_{H}^{\epsilon}(s)-\frac{1}{2} \sigma^{2} \epsilon^{2 \alpha} s\right) d B_{H}^{\epsilon}(s), \\
X_{u}^{\epsilon \alpha}(t)= & X_{u}^{\epsilon \alpha}(0)+\int_{0}^{t} \mu X_{u}^{\epsilon \alpha}(s) d s \\
& +\int_{0}^{t} \frac{\sigma}{2}\left(X_{l}^{\epsilon 1}(0)+X_{u}^{\epsilon 1}(0)\right) \exp \left(\mu s+\sigma B_{H}^{\epsilon}(s)-\frac{1}{2} \sigma^{2} \epsilon^{2 \alpha} s\right) d B_{H}^{\epsilon}(s),
\end{aligned}
$$

or in terms of the Wiener process $W$, we have

$$
\begin{aligned}
X_{l}^{\epsilon \alpha}(t)= & X_{l}^{\epsilon \alpha}(0)+\int_{0}^{t} \mu X_{l}^{\epsilon \alpha}(s) d s \\
& +\alpha \frac{\sigma}{2}\left(X_{l}^{\epsilon 1}(0)+X_{u}^{\epsilon 1}(0)\right) \\
& \times \int_{0}^{t} \varphi^{\epsilon}(s) \exp \left(\mu s+\sigma \alpha \int_{0}^{s} \varphi^{\epsilon}(u) d u-\frac{1}{2} \sigma^{2} \epsilon^{2 \alpha} s+\sigma \epsilon^{\alpha} W(s)\right) d s \\
& +\epsilon^{\alpha} \frac{\sigma}{2}\left(X_{l}^{\epsilon 1}(0)+X_{u}^{\epsilon 1}(0)\right) \\
& \times \int_{0}^{t} \exp \left(\mu s+\sigma \alpha \int_{0}^{s} \varphi^{\epsilon}(u) d u-\frac{1}{2} \sigma^{2} \epsilon^{2 \alpha} s+\sigma \epsilon^{\alpha} W(s)\right) d W(s), \\
X_{u}^{\epsilon \alpha}(t)= & X_{u}^{\epsilon \alpha}(0)+\int_{0}^{t} \mu X_{u}^{\epsilon \alpha}(s) d s \\
& +\alpha \frac{\sigma}{2}\left(X_{l}^{\epsilon 1}(0)+X_{u}^{\epsilon 1}(0)\right) \\
& \times \int_{0}^{t} \varphi^{\epsilon}(s) \exp \left(\mu s+\sigma \alpha \int_{0}^{s} \varphi^{\epsilon}(u) d u-\frac{1}{2} \sigma^{2} \epsilon^{2 \alpha} s+\sigma \epsilon^{\alpha} W(s)\right) d s \\
& +\epsilon^{\alpha} \frac{\sigma}{2}\left(X_{l}^{\epsilon 1}(0)+X_{u}^{\epsilon 1}(0)\right) \\
& \times \int_{0}^{t} \exp \left(\mu s+\sigma \alpha \int_{0}^{s} \varphi^{\epsilon}(u) d u-\frac{1}{2} \sigma^{2} \epsilon^{2 \alpha} s+\sigma \epsilon^{\alpha} W(s)\right) d W(s) .
\end{aligned}
$$

Apply Theorem 8.5.2 in [1] to obtain the unique solution to (3.28) in a form:

$$
\begin{aligned}
X_{l}^{\epsilon \alpha}(t)= & e^{\mu t} X_{l}^{\epsilon \alpha}(0) \\
& +e^{\mu t} \alpha \frac{\sigma}{2}\left(X_{l}^{\epsilon 1}(0)+X_{u}^{\epsilon 1}(0)\right) \\
& \times \int_{0}^{t} \varphi^{\epsilon}(s) \exp \left(\sigma \alpha \int_{0}^{s} \varphi^{\epsilon}(u) d u-\frac{1}{2} \sigma^{2} \epsilon^{2 \alpha} s+\sigma \epsilon^{\alpha} W(s)\right) d s \\
& +e^{\mu t} \epsilon^{\alpha} \frac{\sigma}{2}\left(X_{l}^{\epsilon 1}(0)+X_{u}^{\epsilon 1}(0)\right) \\
& \times \int_{0}^{t} \exp \left(\sigma \alpha \int_{0}^{s} \varphi^{\epsilon}(u) d u-\frac{1}{2} \sigma^{2} \epsilon^{2 \alpha} s+\sigma \epsilon^{\alpha} W(s)\right) d W(s), \\
X_{u}^{\epsilon \alpha}(t)= & e^{\mu t} X_{u}^{\epsilon \alpha}(0) \\
& +e^{\mu t} \alpha \frac{\sigma}{2}\left(X_{l}^{\epsilon 1}(0)+X_{u}^{\epsilon 1}(0)\right) \\
& \times \int_{0}^{t} \varphi^{\epsilon}(s) \exp \left(\sigma \alpha \int_{0}^{s} \varphi^{\epsilon}(u) d u-\frac{1}{2} \sigma^{2} \epsilon^{2 \alpha} s+\sigma \epsilon^{\alpha} W(s)\right) d s
\end{aligned}
$$




$$
\begin{aligned}
& +e^{\mu t} \epsilon^{\alpha} \frac{\sigma}{2}\left(X_{l}^{\epsilon 1}(0)+X_{u}^{\epsilon 1}(0)\right) \\
& \times \int_{0}^{t} \exp \left(\sigma \alpha \int_{0}^{s} \varphi^{\epsilon}(u) d u-\frac{1}{2} \sigma^{2} \epsilon^{2 \alpha} s+\sigma \epsilon^{\alpha} W(s)\right) d W(s) .
\end{aligned}
$$

Then

$$
\begin{aligned}
X_{l}^{\epsilon \alpha}(t)= & e^{\mu t}\left[X_{l}^{\epsilon \alpha}(0)+\frac{\sigma}{2}\left(X_{l}^{\epsilon 1}(0)+X_{u}^{\epsilon 1}(0)\right)\right. \\
& \left.\times \int_{0}^{t} \exp \left(\sigma B_{H}^{\epsilon}(s)-\frac{1}{2} \sigma^{2} \epsilon^{2 \alpha} s\right) d B_{H}^{\epsilon}(s)\right], \\
X_{u}^{\epsilon \alpha}(t)= & e^{\mu t}\left[X_{u}^{\epsilon \alpha}(0)+\frac{\sigma}{2}\left(X_{l}^{\epsilon 1}(0)+X_{u}^{\epsilon 1}(0)\right)\right. \\
& \left.\times \int_{0}^{t} \exp \left(\sigma B_{H}^{\epsilon}(s)-\frac{1}{2} \sigma^{2} \epsilon^{2 \alpha} s\right) d B_{H}^{\epsilon}(s)\right] .
\end{aligned}
$$

Therefore, the approximation fuzzy solution for $\mu \geq 0$ is

$$
\begin{aligned}
X^{\epsilon}(t)= & e^{\mu t} X(0) \\
& +\left\langle\frac{\sigma}{2}\left(X_{l}^{\epsilon 1}(0)+X_{u}^{\epsilon 1}(0)\right) e^{\mu t} \int_{0}^{t} \exp \left(\sigma B_{H}^{\epsilon}(s)-\frac{1}{2} \sigma^{2} \epsilon^{2 \alpha} s\right) d B_{H}^{\epsilon}(s)\right\rangle .
\end{aligned}
$$

For $\mu<0$, we can show that

$$
\begin{aligned}
X_{l}^{\epsilon \alpha}(t)= & X_{l}^{\epsilon \alpha}(0)+\int_{0}^{t} \mu X_{u}^{\epsilon \alpha}(s) d s \\
& +\alpha \frac{\sigma}{2}\left(X_{l}^{\epsilon 1}(0)+X_{u}^{\epsilon 1}(0)\right) \\
& \times \int_{0}^{t} \varphi^{\epsilon}(s) \exp \left(\mu s+\sigma \alpha \int_{0}^{s} \varphi^{\epsilon}(u) d u-\frac{1}{2} \sigma^{2} \epsilon^{2 \alpha} s+\sigma \epsilon^{\alpha} W(s)\right) d s \\
& +\epsilon^{\alpha} \frac{\sigma}{2}\left(X_{l}^{\epsilon 1}(0)+X_{u}^{\epsilon 1}(0)\right) \\
& \times \int_{0}^{t} \exp \left(\mu s+\sigma \alpha \int_{0}^{s} \varphi^{\epsilon}(u) d u-\frac{1}{2} \sigma^{2} \epsilon^{2 \alpha} s+\sigma \epsilon^{\alpha} W(s)\right) d W(s), \\
X_{u}^{\epsilon \alpha}(t)= & X_{u}^{\epsilon \alpha}(0)+\int_{0}^{t} \mu X_{l}^{\epsilon \alpha}(s) d s \\
& +\alpha \frac{\sigma}{2}\left(X_{l}^{\epsilon 1}(0)+X_{u}^{\epsilon 1}(0)\right) \\
& \times \int_{0}^{t} \varphi^{\epsilon}(s) \exp \left(\mu s+\sigma \alpha \int_{0}^{s} \varphi^{\epsilon}(u) d u-\frac{1}{2} \sigma^{2} \epsilon^{2 \alpha} s+\sigma \epsilon^{\alpha} W(s)\right) d s \\
& +\epsilon^{\alpha} \frac{\sigma}{2}\left(X_{l}^{\epsilon 1}(0)+X_{u}^{\epsilon 1}(0)\right) \\
& \times \int_{0}^{t} \exp \left(\mu s+\sigma \alpha \int_{0}^{s} \varphi^{\epsilon}(u) d u-\frac{1}{2} \sigma^{2} \epsilon^{2 \alpha} s+\sigma \epsilon^{\alpha} W(s)\right) d W(s) .
\end{aligned}
$$

The unique solution to (3.30) is of the following matrix form:

$$
X_{l}^{\epsilon \alpha}(t)=X_{l}^{\epsilon \alpha}(0) \cosh (\mu t)+X_{u}^{\epsilon \alpha}(0) \sinh (\mu t)
$$




$$
\begin{aligned}
& +e^{\mu t} \alpha \frac{\sigma}{2}\left(X_{l}^{\epsilon 1}(0)+X_{u}^{\epsilon 1}(0)\right) \\
& \times \int_{0}^{t} \varphi^{\epsilon}(s) \exp \left(\sigma \alpha \int_{0}^{s} \varphi^{\epsilon}(u) d u-\frac{1}{2} \sigma^{2} \epsilon^{2 \alpha} s+\sigma \epsilon^{\alpha} W(s)\right) d s \\
& +e^{\mu t} \epsilon^{\alpha} \frac{\sigma}{2}\left(X_{l}^{\epsilon 1}(0)+X_{u}^{\epsilon 1}(0)\right) \\
& \times \int_{0}^{t} \exp \left(\sigma \alpha \int_{0}^{s} \varphi^{\epsilon}(u) d u-\frac{1}{2} \sigma^{2} \epsilon^{2 \alpha} s+\sigma \epsilon^{\alpha} W(s)\right) d W(s), \\
X_{u}^{\epsilon \alpha}(t)= & X_{l}^{\epsilon \alpha}(0) \sinh (\mu t)+X_{u}^{\epsilon \alpha}(0) \cosh (\mu t) \\
& +e^{\mu t} \alpha \frac{\sigma}{2}\left(X_{l}^{\epsilon 1}(0)+X_{u}^{\epsilon 1}(0)\right) \\
& \times \int_{0}^{t} \varphi^{\epsilon}(s) \exp \left(\sigma \alpha \int_{0}^{s} \varphi^{\epsilon}(u) d u-\frac{1}{2} \sigma^{2} \epsilon^{2 \alpha} s+\sigma \epsilon^{\alpha} W(s)\right) d s \\
& +e^{\mu t} \epsilon^{\alpha} \frac{\sigma}{2}\left(X_{l}^{\epsilon 1}(0)+X_{u}^{\epsilon 1}(0)\right) \\
& \times \int_{0}^{t} \exp \left(\sigma \alpha \int_{0}^{s} \varphi^{\epsilon}(u) d u-\frac{1}{2} \sigma^{2} \epsilon^{2 \alpha} s+\sigma \epsilon^{\alpha} W(s)\right) d W(s) .
\end{aligned}
$$

Then

$$
\begin{aligned}
X_{l}^{\epsilon \alpha}(t)= & X_{l}^{\epsilon \alpha}(0) \cosh (\mu t)+X_{u}^{\epsilon \alpha}(0) \sinh (\mu t) \\
& +e^{\mu t} \frac{\sigma}{2}\left(X_{l}^{\epsilon 1}(0)+X_{u}^{\epsilon 1}(0)\right) \int_{0}^{t} \exp \left(\sigma B_{H}^{\epsilon}(s)-\frac{1}{2} \sigma^{2} \epsilon^{2 \alpha} s\right) d B_{H}^{\epsilon}(s), \\
X_{u}^{\epsilon \alpha}(t)= & X_{l}^{\epsilon \alpha}(0) \sinh (\mu t)+X_{u}^{\epsilon \alpha}(0) \cosh (\mu t) \\
& +e^{\mu t} \frac{\sigma}{2}\left(X_{l}^{\epsilon 1}(0)+X_{u}^{\epsilon 1}(0)\right) \int_{0}^{t} \exp \left(\sigma B_{H}^{\epsilon}(s)-\frac{1}{2} \sigma^{2} \epsilon^{2 \alpha} s\right) d B_{H}^{\epsilon}(s) .
\end{aligned}
$$

Consequently, the approximation fuzzy solution for $\mu<0$ is

$$
\begin{aligned}
X^{\epsilon}(t)= & X(0) \cosh (\mu t)+X(0) \sinh (\mu t) \\
& +\left\langle\frac{\sigma}{2}\left(X_{l}^{\epsilon 1}(0)+X_{u}^{\epsilon 1}(0)\right) e^{\mu t} \int_{0}^{t} \exp \left(\sigma B_{H}^{\epsilon}(s)-\frac{1}{2} \sigma^{2} \epsilon^{2 \alpha} s\right) d B_{H}^{\epsilon}(s)\right\rangle .
\end{aligned}
$$

\section{Conclusions}

We introduced a fuzzy stochastic differential equation with respect to the Liouville form $\mathrm{fBm}$, which has many properties like a long-range dependence. We applied an approximation approach to fractional stochastic integrals and the embedding of classical Itô integral into fuzzy set space. Using the Picard iteration method we studied the existence and uniqueness of the solutions. We proved that the approximate solution converges uniformly to the exact solution.

\section{Acknowledgements}

Not applicable. 
Availability of data and materials

Not applicable.

\section{Competing interests}

The authors declare that they have no competing interests.

\section{Authors' contributions}

The main idea of this paper was proposed by MTM. HJ prepared the manuscript initially and performed all the steps of the proofs in this research. All authors read and approved the final manuscript.

\section{Author details}

'Department of Mathematics, Chabahar Maritime University, Chabahar, Iran. ${ }^{2}$ Department of Applied Mathematics, Cracow University of Technology, ul. Warszawska 24, 31-155, Kraków, Poland.

\section{Publisher's Note}

Springer Nature remains neutral with regard to jurisdictional claims in published maps and institutional affiliations.

Received: 25 June 2019 Accepted: 14 December 2020 Published online: 07 January 2021

\section{References}

1. Allen, E.: Modeling with Itô Stochastic Differential Equations. Springer, Berlin (2007)

2. Alòs, E., Mazet, O., Nualart, D.: Stochastic calculus with respect to fractional Brownian motion with Hurst parameter lesser than 1/2. Stoch. Process. Appl. 86, 121-139 (2000)

3. Alòs, E., Nualart, D.: Stochastic integration with respect to the fractional Brownian motion. Stoch. Stoch. Rep. 75, 129-152 (2002)

4. Carmona, P., Coutin, L., Montseny, G.: Stochastic integration with respect to fractional Brownian motion. Ann. Inst. Henri Poincaré 39, 27-68 (2003)

5. Colubi, A., Dominguez-Menchero, J.S., Lopez-Diaz, M., Ralescu, D.A.: $A D_{E}[0,1]$ representation of random upper semi continuous functions. Proc. Am. Math. Soc. 130, 3237-3242 (2002)

6. Decreusefond, L., Üstünel, A.S.: Stochastic analysis of the fractional Brownian motion. Potential Anal. 10, 177-214 (1998)

7. Diamond, P., Kloeden, P.E.: Metric Spaces of Fuzzy Sets: Theory and Applications. World Scientific, Singapore (1994)

8. Hiai, F., Umegaki, H.: Integrals, conditional expectation, and martingales of multivalued functions. J. Multivar. Anal. 7, 149-182 (1977)

9. Kim, J.H.: On fuzzy stochastic differential equations. J. Korean Math. Soc. 42, 153-169 (2005)

10. Kolmogorov, A.N.: Wienersche Spiralen und einige andere interessante Kurven im Hilbertschen Raum. (German) C. R. (Doklady) Acad. URSS (N.S.) 26, 115-118 (1940)

11. Lakshmikantham, V., Mohapatra, R.N.: Theory of Fuzzy Differential Equations and Inclusions. Taylor \& Francis, London (2003)

12. Malinowski, M.T.: Stochastic fuzzy differential equations with an application. Kybernetika 47, 123-143 (2011)

13. Malinowski, M.T:: Strong solutions to stochastic fuzzy differential equations of Itô type. Math. Comput. Model. 55 , 918-928 (2012)

14. Malinowski, M.T.: Itô type stochastic fuzzy differential equations with delay. Syst. Control Lett. 61, 692-701 (2012)

15. Malinowski, M.T.: Some properties of strong solutions to stochastic fuzzy differential equations. Information Sciences $252,62-80(2013)$

16. Mandelbrot, B.B., Van Ness, J.W.: Fractional Brownian motions, fractional noises and applications. SIAM Rev. 10, 422-437 (1968)

17. Puri, M.L., Ralescu, D.A.: Fuzzy random variables. J. Math. Anal. Appl. 114, 409-422 (1986)

18. Thao, T.H.: An approximate approach to fractional analysis for finance. Nonlinear Anal., Real World Appl. 7(1), 124-132 (2006)

19. Young, L.C.: An inequality of the Hölder type connected with Stieltjes integration. Acta Math. 67, 251-282 (1936)

\section{Submit your manuscript to a SpringerOpen ${ }^{\circ}$ journal and benefit from:}

- Convenient online submission

- Rigorous peer review

- Open access: articles freely available online

- High visibility within the field

- Retaining the copyright to your article

Submit your next manuscript at $\gg$ springeropen.com 